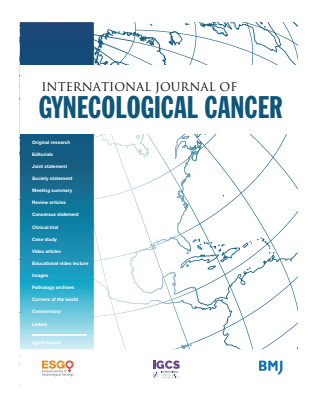

${ }^{1}$ Division of Gynecologic Oncology, Philadelphia, Pennsylvania, USA ${ }^{2}$ Department of Gynecologic Oncology and Reproductive Medicine, MD Anderson Cancer Center, Houston, Texas, USA

\section{Correspondence to} Dr Pedro T Ramirez, Department of Gynecologic Oncology and Reproductive Medicine, MD Anderson Cancer Center, Houston, TX 77230, USA; peramire@mdanderson. org

Received 20 December 2021 Accepted 20 December 2021 Published Online First 17 January 2022

\section{Sinked}

- http://dx.doi.org/10.1136/ ijgc-2021-002544

Check for updates

() IGCS and ESGO 2022. No commercial re-use. See rights and permissions. Published by BMJ.

To cite: Nasioudis D, Ramirez PT. Int J Gynecol

Cancer 2022;32:125-126.

\title{
Is prior conization the way forward to determine surgical approach? The answer is not so simple!
}

\author{
Dimitrious Nasioudis, ${ }^{1}$ Pedro T Ramirez ${ }^{2}$
}

The publication of the LACC (Laparoscopic Approach to Cervical Cancer) trial, ${ }^{1}$ demonstrating worse disease-free and overall survival in patients undergoing minimally invasive radical hysterectomy, led to a change in practice recommendations with open radical hysterectomy as the new standard of care. ${ }^{2}$ Similarly, a number of studies have shown a significant decrease in the rate of minimally invasive radical hysterectomy in the USA since the publication of the LACC trial. ${ }^{34}$ Hypotheses for the inferior outcomes with minimally invasive surgery include tumor contamination, use of a uterine manipulator, and/or increased tumor dissemination and implantation as a result of pneumoperitoneum. Data from retrospective studies suggest that the absence of a gross cervical lesion, omission of a uterine manipulator, and performance of vaginal protective maneuvers could potentially mitigate the increased risk of relapse observed with minimally invasive radical hysterectomy. ${ }^{5-7}$

In this issue, Chacon et al ${ }^{8}$ report on a sub-analysis of the SUCCOR study examining the effect of cervical conization prior to radical hysterectomy in patients with International Federation of Gynecology and Obstetrics (FIG0) 2009 stage IB1 cervical cancer. Authors performed propensity score matching in an attempt to minimize potential bias and adjust for differences in adjuvant treatment and risk factors such as presence of nodal metastases, depth of stromal invasion, and lymphovascular invasion. The authors reported that cervical conization was associated with a $65 \%$ reduction in the risk of relapse and a $75 \%$ reduction in the risk of death. Based on exploratory subgroup analyses, differences in oncologic outcomes were mainly driven by patients who underwent minimally invasive radical hysterectomy. These results are in accordance with prior smaller retrospective studies demonstrating better oncologic outcomes for patients who previously underwent conization. ${ }^{9}$

The authors of the SUCCOR study should be applauded for bringing together 126 participating institutions to answer clinically relevant questions. While these results are thought provoking, there are significant inherent limitations, as acknowledged by the authors. First, final tumor size determination following conization may be challenging and was not standardized. It has been previously demonstrated that measurement of tumor size in patients with cervical cancer is prone to significant discrepancies, considering that there is a lack of agreement among pathologist as to how to determine tumor size when patients have had a conization, and that residual disease is subsequently found in the hysterectomy specimen. ${ }^{10}{ }^{11}$ This is also compounded by certain scenarios that hamper consistency in the definition of tumor size. These include the presence of multifocal disease on the cervix, use of tumor 'dimension' versus 'diameter', or validity of imaging versus physical examination, particularly when there is discrepancy between these. One should also note that in the current study by Chacon et al, ${ }^{8}$ overall tumor size appeared to be larger in the non-conization group (median 19 vs $12 \mathrm{~mm}$ ). Even among patients with tumors $<2 \mathrm{~cm}$, differences in tumor size (1.8 vs $0.5 \mathrm{~cm}$ ) could account for the observed difference in relapse rates. This difference in tumor size when comparing groups should be highlighted and is an important point that is often missed in discussions. It is not uncommon that in studies that propose conization is associated with lower risks of recurrence, tumor size is generally larger in the group that did not undergo conization-thus raising the point that it is not surprising that in the group where no conization was performed, the recurrence rates were expectedly higher.

Other notable factors to be considered are the fact that in nearly $50 \%$ of patients in both groups in this study, ${ }^{8}$ the procedure was performed by minimally invasive surgery and colpoprotective maneuvers were performed in $18.7 \%$ of patients in both groups (conization vs no conization). This highlights a point of caution in how these results are interpreted as these findings may certainly impact oncologic outcomes overall. In addition, as the authors of the current study recognize, there was no evaluation of patients with stage IB1 disease based on microscopic disease versus gross tumor on cervical specimen. Is it possible that patients who underwent conization had by default smaller, potentially clinically occult tumors? 


\section{Editorial}

In other words, was performance of a conization procedure a surrogate marker of less aggressive biologic behavior?

Furthermore, it is unclear what the indications of the conization procedures were; this is particularly relevant as $80.7 \%$ of patients had residual disease in the final hysterectomy specimen, thus leaving readers wondering as to whether conizations were performed on gross visible tumor. Similarly, the decision on the approach to surgery (open vs minimally invasive) is not well documented. Lastly, when considering recurrence rates or diseasefree survival as a primary objective of any study, standardization of surveillance techniques is extremely important and, in this study, it was not specified as to whether recurrences were detected by physical examination, imaging studies, or by pathologic diagnosis.

Some might argue that an ideal strategy moving forward is to design a prospective randomized trial comparing open and minimally invasive surgery in patients who have undergone prior conization. There are several items to consider that may taper that enthusiasm. First, when evaluating patients in the LACC trial ${ }^{1}$ who had tumors $<2 \mathrm{~cm}$, the recurrence rate in the open surgery group was $0.7 \%$ and in the minimally invasive group was $3.3 \%$-thus highlighting the fact that in order to detect a difference in recurrence rates in these two approaches in this patient population, the number of patients would need to be exceedingly high. Additionally, given the results of the recently published ConCerv trial ${ }^{12}$ demonstrating the safety of conservative management in patients with low-risk cervical cancer, it is doubtful that in the future patients with tumors $<2 \mathrm{~cm}$ will even require a radical hysterectomy.

It should be highlighted that this is not a study aiming to answer the question as to whether performing a conization ultimately equates to safety of a minimally invasive surgical approach. That is not the question explored in this study and should not be misinterpreted as such. It should also be highlighted that current guidelines do not support the performance of conization in the setting of grossly invasive cervical cancer to reduce tumor burden and thus proceed with a minimally invasive approach. As the authors suggest, results of the present study should be cautiously interpreted. Open radical hysterectomy remains the current standard of care for all patients with cervical cancer, and minimally invasive techniques, even following conization, should only be considered in the context of research studies. ${ }^{13}$ Two ongoing randomized trials are currently recruiting patients, ${ }^{14}{ }^{15}$ and until their results are published, data derived from retrospective studies should only be regarded as hypothesis generating.

Twitter Pedro T Ramirez @pedroramirezMD

Contributors Both authors contributed to the writing of the article.
Funding The authors have not declared a specific grant for this research from any funding agency in the public, commercial or not-for-profit sectors.

Competing interests None declared.

Patient consent for publication Not applicable.

Ethics approval This study does not involve human participants.

Provenance and peer review Commissioned; internally peer reviewed.

\section{REFERENCES}

1 Ramirez PT, Frumovitz M, Pareja R, et al. Minimally invasive versus abdominal radical hysterectomy for cervical cancer. N Engl J Med 2018;379:1895-904.

2 National Comprehensive Cancer Network. NCCN clinical practice guidelines in oncology(NCCN guidelines), 2021. Available: https:// www.nccn.org/professionals/physician_gls/pdf/cervical.pdf

3 Charo LM, Vaida F, Eskander RN, et al. Rapid dissemination of practice-changing information: a longitudinal analysis of real-world rates of minimally invasive radical hysterectomy before and after presentation of the LACC trial. Gynecol Oncol 2020;157:494-9.

4 Matsuo K, Mandelbaum RS, Klar M, et al. Decreasing utilization of minimally invasive hysterectomy for cervical cancer in the United States. Gynecol Oncol 2021;162:43-9.

5 Chiva L, Zanagnolo V, Querleu D, et al. SUCCOR study: an international European cohort observational study comparing minimally invasive surgery versus open abdominal radical hysterectomy in patients with stage IB1 cervical cancer. Int J Gynecol Cancer 2020;30:1269-77.

6 Kampers J, Gerhardt E, Sibbertsen P, et al. Protective operative techniques in radical hysterectomy in early cervical carcinoma and their influence on disease-free and overall survival: a systematic review and meta-analysis of risk groups. Arch Gynecol Obstet 2021;304:577-87.

7 Uppal S, Gehrig PA, Peng K, et al. Recurrence rates in patients with cervical cancer treated with abdominal versus minimally invasive radical hysterectomy: a multi-institutional retrospective review study. J Clin Oncol 2020;38:1030-40.

8 Chacon E, Manzour N, Zanagnolo V. SUCCOR cone study: conization before radical hysterectomy. Int J Gynecol Cancer 2022;32:117-24.

9 Casarin J, Bogani G, Papadia A, et al. Preoperative conization and risk of recurrence in patients undergoing laparoscopic radical hysterectomy for early stage cervical cancer: a multicenter study. $J$ Minim Invasive Gynecol 2021;28:117-23.

10 Salvo G, Odetto D, Pareja R, et al. Revised 2018 International Federation of Gynecology and Obstetrics (FIGO) cervical cancer staging: a review of gaps and questions that remain. Int J Gynecol Cancer 2020;30:873-8.

11 Salvo G, Odetto D, Saez Perrotta MC, et al. Measurement of tumor size in early cervical cancer: an ever-evolving paradigm. Int $J$ Gynecol Cancer 2020;30:1215-23.

12 Schmeler KM, Pareja R, Lopez Blanco A, et al. ConCerv: a prospective trial of conservative surgery for low-risk early-stage cervical cancer. Int J Gynecol Cancer 2021;31:1317-25.

13 NICE. Minimally invasive radical hysterectomy for early stage cervical cancer. National Institute for Health and Care Excellence. Available: www.nice.org.uk/guidance/ipg686

14 Falconer H, Palsdottir K, Stalberg K, et al. Robot-assisted approach to cervical cancer (RacC): an international multi-center, open-label randomized controlled trial. Int J Gynecol Cancer 2019;29:1072-6.

15 Chao X, Li L, Wu M, et al. Efficacy of different surgical approaches in the clinical and survival outcomes of patients with early-stage cervical cancer: protocol of a phase III multicentre randomised controlled trial in China. BMJ Open 2019;9:e029055. 